\title{
Field Handling and Anti-Rabies Vaccine Efficacy
}

\author{
"OLADOKUN, A.T., ${ }^{2}$ MESEKO, C.A., ${ }^{3}$ LAZARUS, D.D. ${ }^{1}$ SATI, L.D., ${ }^{4} E K O N G$ P.S., ${ }^{3}$ NWOSUH, C.I. \\ ${ }^{1}$ Quality Control Division, ${ }^{2}$ Viral Research Division, ${ }^{3}$ Viral Vaccine Production Division ${ }^{4}$ Central Diagnostic Division, National Veterinary \\ Research Institute Vom *Corresponding Author: tinagnes80@yahoo.com, +234-8036794244
}

\begin{abstract}
SUMMARY
Anti-rabies vaccine for dogs, earlier procured in 2008 from National Veterinary Research Institute, Vom was received for re-evaluation from the field. The field originated and reference samples of the same production batch (04/2008) were inoculated into 3-week old mice intracerebrally for comparison and observed daily for 21 days. There were no signs of illness, paralysis or death in the groups of mice inoculated with the field vaccine. Whereas deaths were observed between days 7 and 18 post inoculation in the groups inoculated with the reference sample, an indication of live rabies vaccine virus. Animals in the control group remained healthy throughout the period of observation. This study shows that not all vaccines produced under Good Laboratory Practice and passed are biologically viable following exposure to field conditions due to poor handling and other factors. For efficiency of immunization therefore, the quality of anti-rabies vaccine must be ensured by maintaining an effective cold chain on storage between the time of production, procurement and usage in the field.
\end{abstract}

KEYWORDS: Anti rabies vaccine, Field handling, Efficacy, Re-evaluation.

\section{INTRODUCTION}

Dog remains the most important agent of transmission of rabies to humans in Africa and Asia (Warrell and Warrell 1988; CDC, 2009; Benka, 2009). The administration of human rabies immunoglobulin (HRIG) combined with post exposure vaccination can neutralize the virus before it invades the nervous system in the case of human victim thus effectively preventing clinical manifestation of rabies and death in man (Jackson et al., 2003). However, rabid dogs would continue to infect human population with the disease in its most virulent form because of continuous circulation of the virus in susceptible population. This may result in diverse strains that can evade the immune systems of both man and animal. Thus, rabies, though a preventable zoonosis, is yet to be effectively controlled and its true impact is largely underestimated (Coleman et al., 2004).

The importance of rabies control in dogs by vaccination cannot be overemphasised. This effective means of rabies prevention has however been hampered by inadequate resources, low public awareness, poor surveillance, inadequate research, poor dog vaccination coverage and poor compliance in resource poor countries. With an estimated 55,000 human deaths reported annually, mainly in Africa and Asia, rabies control demands more seriousness of an emergency disease on the part of policy makers and researchers (Knobel et al., 2005; CDC, 2009)

Nigerian Governments at all levels and NonGovernmental Organisations have made immense contributions through the annual antirabies vaccination campaigns in dogs and cats. Such State and Local Government Authorities mostly procure anti-rabies vaccines which are administered either at a subsidized rate or free of charge to dogs within their localities. These vaccines are often sourced directly from National Veterinary Research Institute (NVRI), Vom (the only veterinary vaccine producing institution in the country) and distributed through a chain until they get to the field. However, these networks of distribution do not always ensure the maintenance of cold chain. Thus the immunogenicity/viability of the vaccines at the point of use cannot be guaranteed. Following complaint from the field, this study was carried out to re-assess the viability of rabies vaccine sourced from NVRI Vom. 


\section{Materials and Methods Anti-rabies Vaccine}

Two vials of anti-rabies vaccine produced by NVRI (Batch 04/2008) and procured by Shelleng Local Government Area of Adamawa state were received by the Quality Control Division, NVRI, Vom with a request for reevaluation. The vaccines had been with the client for about 4 months prior to submission. Handling of these vaccines was believed to have been compromised in the field, and the authority wished to revalidate its potency before being issued for anti rabies campaign in the Local Government Area. These vaccines were tested alongside the reference samples of the same production batch.

\section{Laboratory Animals}

One hundred and twenty white albino mice (3-4 weeks old) were obtained from the Small Animal Experimental Unit, Central Diagnostic Division, NVRI, Vom and 96 of these were assigned to groups of 8 each (F1-F5 for the field originated; $\mathrm{R} 1-\mathrm{R} 5$ for the reference vaccines and C1\&C2 for negative controls). The mice were given pelletized feed and water ad libitum and allowed to stabilize for 24 hours before being used.

\section{Sample Preparation}

Two vials of anti rabies vaccines received from the field were reconstituted with $2.5 \mathrm{ml}$ of PBS (pH 7.4) each. The reconstituted vaccines from the two vials were pooled and kept on ice. A ten fold serial dilution of the reconstituted vaccine was made in Bijou bottles containing sterile PBS (pH 7.4) up to $10^{-5}$ dilutions. The same procedure was repeated for two vials of vaccines from the reference sample.

\section{Mice inoculation}

Starting from the highest $\left(10^{-5}\right.$ dilution) to the lowest dilution $\left(10^{-1}\right), 0.03 \mathrm{ml}$ of each dilution was inoculated into 8 mice using a $0.5 \mathrm{ml}$ tuberculin syringe fitted with $27 \mathrm{G} \mathrm{X} 1 / 2$ " needle intracerebrally. Eight un-inoculated mice were kept alongside to serve as negative control. This was also repeated for the reference samples. The mice were observed daily for signs of illness, paralysis and/or death for 21 days and observations recorded. The $\mathrm{LD}_{50}$ was calculated using Spearman Karber formula; $\log \mathrm{LD}_{50}=\mathrm{X}_{\mathrm{o}}$ $\mathrm{d} / 2+\mathrm{d}\left(\sum \mathrm{r}_{\mathrm{i}} / \mathrm{n}_{\mathrm{i}}\right)$ where:

$X_{o}-\log _{10}$ of the reciprocal of the lowest dilution at which all the test mice died

$\mathrm{d}-\log _{10}$ of the dilution factor (i.e. the difference between the log dilution intervals)

$\mathrm{n}_{\mathrm{i}}$ - number of test mice used at each dilution (after discarding accidental losses)

$\mathrm{r}_{\mathrm{i}}$ - number of dead mice (out of $\mathrm{n}_{\mathrm{i}}$ )

$\sum \mathrm{r}_{\mathrm{i}} / \mathrm{h}_{\mathrm{i}}$ - sum of the proportion of positive tests beginning at the lowest dilution showing 100 rhortality (positive result)

\section{RESULTS}

Throughout the period of observation, there were no sign of illness, paralysis or death in all the groups of mice inoculated with the vaccine from the field. Death were observed between days 7 and 18 post inoculation in the groups inoculated with the reference sample of the same batch of vaccine, while the controls within this group remained healthy throughout the period of observation (Fig 1). The summary of the observations is as shown in Table I.

Figure 1: Proportion of dead to live mice against days post inoculation of the reference and the field vaccine at dilution $10^{-2}$

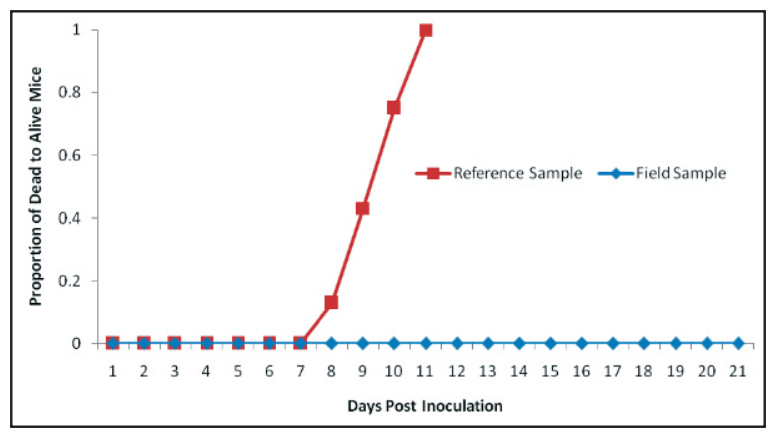

TABLE I: Proportion of dead to inoculated mice at the different dilutions of the reference compared with the field vaccine

\begin{tabular}{|cc|cc|cc|}
\hline & & \multicolumn{2}{|c|}{ Reference Sample } & \multicolumn{2}{c|}{ Field Sample } \\
\hline Dilution & $\mathrm{n}_{\mathrm{i}}$ & $\mathrm{r}_{\mathrm{i}}$ & $\mathrm{P}=\left(\mathrm{r}_{\mathrm{i}} / \mathrm{ni}\right)$ & $\mathrm{r}_{\mathrm{i}}$ & $\mathrm{P}=\left(\mathrm{r}_{\mathrm{i}} / \mathrm{n}_{\mathrm{i}}\right)$ \\
\hline $10-^{-}$ & 8 & 8 & 1 & 0 & 0 \\
$10-^{2}$ & 8 & 8 & 1 & 0 & 0 \\
$10-^{3}$ & 8 & 6 & 0.75 & 0 & 0 \\
$10{ }^{-}$ & 8 & 2 & 0.25 & 0 & 0 \\
$10-{ }^{5}$ & 8 & 0 & 0 & 0 & 0 \\
\hline
\end{tabular}

$n_{i}=$ No. of mice inoculated per dilution, $r_{i}=$ No. of dead mice,

$\mathrm{P}=$ Proportion of dead to inoculated mice 


\section{Calculations}

Using the Spearman Karber formula, $\log \mathrm{LD}_{50}=$ Xo - d $/ 2+\mathrm{d}\left(\sum \mathrm{ri} / \mathrm{ni}\right)$

$\log \mathrm{LD}_{50} \quad=(-2-1 / 2+1(1+0.75+0.25+0)$ $=-3.5$

Titre $=\log \mathrm{Ld}_{50} 3.5 / 0.03 \mathrm{ml}$ of the vaccine $=10-^{3.5}$ per $0.03 \mathrm{ml}$

The OIE recommends vaccine batch with a titre of $10^{-3.3}$ per $0.03 \mathrm{ml}$ for use but an additional 0.5 $\log$ at production, is required to accommodate loss due to storage. The titre of the reference sample of the vaccine $10^{-3.5}$ is consistent with the standard since the vaccine had been in storage for almost one year which is the producer's recommended shelf life. In this experiment, the reference vaccine was viable and potent being able to cause death in mice that were inoculated. However, the vaccine from the field appears to have lost viability and was unable to initiate illness, paralysis or death when inoculated intracerebrally in mice.

\section{DISCUSSION}

Dog rabies has been recognised since the advent of human civilization (Wilkinson, 2002), and is responsible for a most severe human suffering and death. Despite concerted effort at control, domestic dogs still pose the greatest public health hazard with regards to rabies (Belotto et al., 2005) in the developing countries. As revealed in this study, biologically viable and potent rabies vaccine may lose viability and potency due to poor handling and such vaccine is ineffective against rabies control and management. In countries with canine rabies, the disease control/eradication policy has always been mass vaccination for the elimination of the virus in the major reservoir host. In Nigeria, though rabies has been responsible for human death and suffering (Fagbami et al., 1981), vaccination has been poorly implemented with low coverage and compliance rates similar to what obtain in most developing countries. In developed countries like the USA, Canada and Western Europe, urban rabies have been successfully controlled with strict vaccination policy of domestic dogs and cats (WHO, 2005). Available records in the Institute's Veterinary Clinic, which serve as the main repository for rabies and rabies related activities for Nigeria showed that out of a total of 2,360 dogs suspected of rabies between 19992008 , only $171(7.2 \%)$ had vaccination records, the effectiveness of such vaccination may even be doubted in view of the present study. According to the WHO, comprehensive control measure for rabies must include mass vaccination of dogs through campaigns and by continuous vaccination of young dogs (WHO, 2010).

Controlling rabies has been the mandate of governments; vaccination programme for dogs generally consisted of periodic visits by a government vaccination team on campaigns to communities. This may have some effect on reducing rabies, when the quality of the vaccine can be guaranteed. The current work shows that the efficacy of anti-rabies vaccines currently in use and their effectiveness in the control of rabies should be regularly checked. Rabies is preventable by breaking the cycle of transmission in dog to dog and man with immunogenic vaccines that are not compromised by poor handling on the field. Reducing the risk of human exposure and safe guarding public health is hinged on these recommendations.

\section{ACKNOWLEGDEMENT}

The authors acknowledge the contributions of staff of Quality Control, Viral Research and Viral Vaccine Production Divisions of National Veterinary Research Institute, Vom.

\section{REFERENCES}

BELOTTO, A., LEANES, L. F., SCHNEIDER, M. C., TAMAYO, H. and CORREA, E. (2005): Overview of rabies in the Americas.Virus Res., 111(1):5-12.

BENKA VALERE (2009): Nepal animal birth control/anti-rabies research. Proceedings of Animal Welfare in Veterinary Medical Education and Research Nov 9-11, 2009. The Kellogg Hotel and Conference Centre at Michigan State University, Michigan USA

CENTERS FOR DISEASE CONTROL and PREVENTION 1600 Clifton Rd. Atlanta, GA 30333, USA (2009) http://www.cdc.gov/Features/Rabies/ September 25, 2009

COLEMAN, P. G., FEVRE, E. M. and CLEVELAND, S. (2004): Estimating the public health impact of rabies. Emerg Infet Dis., 10(1):140-2.

FAGBAMI, A. H., ANOSA, V.O. and EZEBURIO, E. O. (1981): Hospital records of human rabies and anti-rabies prophylaxis in Nigeria, 1969-1978. 
Transactions of the Royal Societies of Tropical Medicine and Hygiene, 75: (6) 872-876 for the year 1998. WHO/CDS/CER/APH99.6, Geneva, Switzerland.

JACKSON, A. C., WARRELL, M. J., RUPPRECHT, C. E., ERTL, H. C., DIETZSCHOLD, B., O'REILLY, M., LEACH, R. P., FU, Z. F., WUNNER, W. H., BLECK, T. P. and WILDE, H. (2003): Management of rabies in humans. Clin Infect Dis., 36(1):60-3

KNOBEL, D. L., CLEAVELAND, S., COLEMAN, P. G., FEVRE, E. M., MELTZER, M. I., MIRANDA, M. E., SHAW, A., ZINSSTAG, J. and MESLIN, F. X. (2005): Re-evaluating the burden of rabies in Africa and Asia. Bull World Health Organ.,
83(5):360-8

WARRELL, D. A. and WARRELL, M. J. (1988): Human rabies and its prevention: an overview. Rev Infect Dis. 1092:131-4.

WILKINSON, L. (2002): History. In: Rabies. A. C. Jackson and W. H. Wunner, Eds. New York: Academic Press: 1-22

WORLD HEALTH ORGANIZATION (2005): Expert consultation on rabies, Geneva, 5-8 October 2004(TRS 391). Available: www.who.int/rabies/animal/excerpt

WORLD HEALTH ORGANIZATION (2010): Rabies. www.who.int/zoonoses/diseases/-rabies/en/June 14th 2010 